\title{
PRESENCIA SOCIAL DE MUJERES DE ZONAS RURALES EN LAS REDES SOCIALES
}

\section{RURAL WOMEN'S SOCIAL PRESENCE IN SOCIAL NETWORKS}

\author{
Dra. Luisa Vega Caro \\ luiveg@us.es \\ Dra. Olga Buzón García \\ obuzon@us.es
}
Facultad de Ciencias de la Educación. Universidad de Sevilla.
Departamento de Didáctica y Organización Educativa
Calle Pirotecnia S/N. C.P. 41013. Sevilla (Spain)

En este trabajo analizamos la presencia social de las mujeres de zonas rurales de la provincia de Sevilla en las redes sociales de Internet a través de tres categorías: afecto, interacción y cohesión para conocer el impacto que tienen sobre ellas.

Se aplica una escala de tipo Likert a 478 mujeres de entornos rurales con edades comprendidas entre 18 y 65 años. Los resultados muestran que la presencia social en redes sociales virtuales está definida principalmente por el componente social y comunicativo. En este sentido las mujeres afirman sentirse integradas y escuchadas por otras personas en su red social.

Palabras claves: Presencia Social, redes sociales, mujer rural, estudios de las mujeres.

This paper analyzes the social presence of women in rural areas of the province of Seville on social networks between three categories: affection, interaction and cohesion to understand the impact they have on them.

Likert scale was applied to 478 women from rural areas aged between 18 and 65 years old. The results show that social presence in virtual social networks is mainly defined by the social and communicative component. In this sense, women say they feel integrated and heard by others in their social network.

Key words: Social Presence, Social Networks, Rural Women, Women's Studies.

Píxel-Bit. Revista de Medios y Educación. No 48 Enero 2016. ISSN: 1133-8482. e-ISSN: 2171-7966. doi: http://dx.doi.org/10.12795/pixelbit.2016.i48.10 


\section{Introducción.}

Este trabajo presenta los resultados de una investigación sobre la inclusión digital de las mujeres rurales en las redes sociales, cuyo propósito ha sido conocer el grado de presencia social que existe dentro de las redes sociales online que usan las mujeres.

A lo largo de los últimos años, las diferentes redes sociales existentes en internet han experimentado una gran transformación, convirtiéndose en una herramienta ampliamente utilizada para facilitar y mejorar los procesos de interacción social entre las personas (Limious \& Smith, 2010; Uzunboylu, Bicen \& Cavus, 2011), los cuales, están formados por una serie de dimensiones, como es el caso de la presencia social, que hace posible una mayor comunicación y cohesión entre las personas que interactúan a través de las redes sociales de internet.

Según las aportaciones de Garrison y Anderson (2005) y Kreijns (2004), la presencia social es uno de los elementos fundamentales que interviene en la comunicación online. La misma, hace referencia a la capacidad que tenemos las personas para proyectarnos de manera social y emocional (Garrison \& Archer, 2001). Su ausencia dificulta la capacidad para expresar opiniones, desacuerdos, compartir puntos de vista o aceptar ayuda. La presencia social es un concepto importante para entender el contexto social en el que se relacionan las personas, ya que afecta al tipo y grado de interacción creado a través del ordenador así como el escenario que se produce en las redes sociales. En este sentido, Kreijns (2004) afirma que la presencia social fomenta y enriquece las interacciones sociales en línea, al ser el vehículo principal del aprendizaje social, entendiendo dicho concepto como el proceso por el que las mujeres que están interconectadas entre sí a través de la red, sienten una interdependencia afectiva, actitudinal y conductual con otras personas que conllevan a reducir la sensación de soledad o aislamiento de la sociedad, sintiéndose parte de ésta. Para ello, en primer lugar, presentamos diferentes definiciones que se han dado respecto al concepto de presencia social tanto a nivel internacional como nacional, al objeto de dar a conocer mejor el término. En segundo lugar, reflejamos la importancia que tiene este concepto en el estudio de las redes sociales. También damos a conocer los resultados obtenidos tras la aplicación de la escala con mujeres de la provincia de Sevilla y, finalizamos el artículo con un apartado de discusión y conclusiones donde se realiza el correspondiente contraste de los hallazgos encontrados en relación con los diversos estudios e investigaciones presentadas.

\subsection{El concepto de presencia social.}

El término de presencia social se introdujo en 1976 por Short, Williams y Christie en el campo de la psicología social y de la comunicación, cuando el uso de internet estaba comenzando. Estos autores sostienen que los medios difieren en su capacidad de transmitir presencia social, entendiendo ésta como la percepción que tienen los que interactúan en la comunicación con relación a la presencia social y psicológica que experimentan durante la situación de comunicación, es decir, la presencia social se interpreta como el grado en que una persona es percibida como real (Richardson \& Swan, 2003). Estamos ante un tema complejo que debe ser estudiado de diversas formas, teniendo en cuenta factores como la comunicación, interacción y el aprendizaje. 
Estudios como los de Biocca, Harms y Burgoon (2003) definen la presencia social como el grado de apercibimiento, atención localizada, comprensión afectiva y de contenidos, e interdependencia afectiva y conductual con la otra persona, es decir, es el grado en que se percibe a una persona en el resultado de las interacciones personales en la comunicación mediada (Gunawardena, 2003). Respecto al aprendizaje online, éste tiene la capacidad de transmitir algunas comunicaciones en dos vías que propician la intimidad y cercanía para establecer un clima de confianza entre los participantes, de modo que se sientan parte de la comunidad de aprendizaje.

Kreijns (2004), por su parte, define la presencia social como el grado de sensación psicológica en la que existe la ilusión de que el otro, en la comunicación, es una persona real. Se refiere también a la habilidad de los participantes de proyectarse asimismos social y emocionalmente como personas reales, es decir, con su personalidad plena, a través del medio de comunicación utilizado (Garrison, Anderson \& Archer, 2000). En esta línea, destacan varias investigaciones que han analizado las interacciones y procesos de aprendizaje en foros online, indagando en los componentes afectivos y de cohesión (Anderson, Rouke, Garrison \& Acher, 1999; Garrison \& Acher, 2001; Rourke, Anderson, Garrison \& Acher, 2001) a través de un modelo de análisis basado en tres dimensiones básicas: presencia cognitiva, didáctica y presencia social.

- La presencia cognitiva: es entendida como el punto por el cual se es capaz de construir significados mediante la reflexión continua en una comunidad de investigación.

La presencia docente: es definida como la acción de diseñar, facilitar y orientar los procesos cognitivos y sociales, con el objetivo de obtener resultados educativos personalmente significativos y de valor docente.

La presencia social: abarca todas aquellas interacciones que están enfocadas a fomentar la creación de una dinámica grupal, ya que se promueven las relaciones sociales y se expresan emociones. Esta dimensión social, al ser considerada uno de los elementos fundamentales dentro del aprendizaje online, fue analizada partiendo de tres categorías:

Afectiva: tiene que ver con la expresión de las emociones o el sentido del humor. La expresión emocional es entendida como la capacidad y la confianza para expresar los propios sentimientos a través de la red.

Interactiva: está relacionada con la continuación de un mensaje, citas de otros o formulación de preguntas. Es todo aquello que está relacionado con el conocimiento y comprensión mutua de las diferentes contribuciones que se realizan de forma online. Se trata de una categoría muy útil para conocer el tipo de motivación que se tiene, al objeto de seguir manteniendo a lo largo del tiempo el contacto con las otras personas con las que interactuamos.

Cohesión de grupo: son todas aquellas actividades que realizamos en la red que permiten crear y mantener un sentido de compromiso de grupo. La construcción de la cohesión y el sentido de pertenencia en este contexto es muy importante, ya que permite que la persona no se sienta como un ser aislado sino parte de una comunidad. Esta categoría se basa en la participación y en la empatía.

Tu y McIsaac (2002), en su estudio sobre el impacto de la presencia social en la interacción online, argumentaron el resultado de una mezcla de métodos de estudio que 
han realizado sobre la presencia social e identificaron como dimensiones las siguientes:

Contexto social: se construye a partir de las características de las personas y sus percepciones con respecto a los entornos virtuales. Está relacionado con la familiaridad y relaciones de confianza creadas con las personas con las que se interactúa.

Comunicación en línea: hace referencia a la frecuencia de la comunicación y calidad de ésta. En la comunicación en línea, el lenguaje usado y las aplicaciones que se le da, es esencial para un aprendizaje colaborativo. Diversos estudios (Garramone, Harris \& Anderson, 1986; Perse, Burton, Kovner, Lears, \& Sen, 1992), han manifestado que existe una relación positiva entre la presencia social y la percepción de los estudiantes sobre su experiencia informática.

Interactividad: incluye la comunicación activa y los estilos de comunicación que se utilizan, tales como tiempo de respuesta, los estilos de comunicación, los tipos de tareas, los temas y el tamaño de los grupos. Cuando se espera una respuesta inmediata y no se recibe, se crea una sensación de interactividad que hace disminuir el nivel de presencia social (Gunawardena 1995). En otras palabras, la interactividad implica las diferentes estrategias que se utilizan para estimular la presencia social.

Privacidad: viene determinado por la percepción sobre la intimidad y privacidad del sistema. La sensación de privacidad se refiere a la percepción de la intimidad psicológica, mental o cultural. A menos intimidad, se produce una disminución de la percepción de la presencia social. La privacidad del sistema se refiere a la seguridad actual de las redes sociales, como considerar la posibilidad de que alguien externo pueda leer o volver a enviar un mensaje sin consentimiento. En un estudio realizado sobre la comunicación mediada por ordenador donde se evaluó la privacidad de las estás tecnologías, Kerr y Hiltz (1982) descubrieron que las personas con un mejor conocimiento de los sistemas informáticos siempre percibían la privacidad baja, debido a que los sistemas no son seguros al cien por cien.

Serrano (2005), en su estudio sobre la evaluación de los indicadores de la presencia social y apoyado en las investigaciones de Garrison, Anderson y Archer (2000) y Rourke et al. (2001), presentó como indicadores de la presencia social los siguientes: a) Afectivo: relacionado con la identidad del propio grupo y la motivación de participar en él y b) Cohesión: es la organización que se establece dentro del propio grupo y el cumplimiento de los objetivos de aprendizaje. Tales mecanismos de motivación, comunicación, participación e interactividad fueron considerados como los principales indicadores para mejorar la participación, la interactividad entre la comunidad en línea y el establecimiento de lazos entre los miembros del grupo.

Estudios más recientes como los de Agut, Grandío y Lozano (2011), estudian la presencia social en entornos virtuales de aprendizaje, realizando la adaptación al español de la medida de la presencia social de las mentes en red de Harms y Biocca (2004). Toman como referencia las diferentes dimensiones de los estudios de Garrison, Anderson y Archer (2000), quienes consideran que las personas se sienten interconectadas a través de interfaces de comunicación en red. Entienden la presencia social, como el grado de apercibimiento, 
atención localizada, comprensión afectiva y de contenidos, e interdependencia afectiva y conductual con otra persona. Desde esta consideración es percibida como un constructo compuesto por seis dimensiones: a) copresencia, que alude al grado en que la persona siente que no está sola y aislada en la red; b) interdependencia afectiva percibida, entendida como la capacidad para comprender el estado emocional y actitudinal del interlocutor/a; c) interdependencia conductual percibida, alude al grado en que la conducta de los interlocutores se afecta mutuamente; d) comprensión percibida del mensaje, es la capacidad para comprender el contenido de los mensaje de los demás; e) dedicación atencional, que hace referencia a la cantidad de atención que los interactuantes en la red se dedican mutuamente y f) comprensión afectiva percibida, entendida como la capacidad para comprender el estado emocional y las actitudes de la otra persona con la que interactuamos.

En este estudio, consideramos que la presencia social es un concepto muy complejo por la diversidad de dimensiones que lo caracterizan, pero necesario para entender el contexto social en el que se relacionan las mujeres de zonas rurales, ya que afecta al tipo y grado de interacción creada a través del ordenador $y$, concretamente, a través de las redes sociales. La importancia de esta investigación radica en que a partir del modelo para el análisis de las interacciones en entornos virtuales (Anderson et al., 1999; Garrison, Anderson \& Archer, 2001; Rourke et al, 2001; Agut et al., 2011), en este trabajo analizamos la presencia social de las mujeres sevillanas en las redes sociales de Internet a través de tres categorías: afecto, interacción y cohesión algo totalmente novedoso en estos tipos de entornos.

\subsection{La presencia social de las mujeres en el uso de las redes sociales.}

Es necesario prestar atención al papel que juegan las redes sociales utilizadas por las mujeres de las zonas rurales de la provincia de Sevilla, ya que éstas son conceptualizadas por autoras como Santos (2011), como entornos virtuales que promueven los sentimientos de cohesión y compromiso con las otras personas con las que interactúan. Asimismo, estimulan la participación social de sus miembros en las diferentes actividades sociales organizadas dentro de la comunidad que se va creando. En este contexto, es importante conocer el tipo y grado en que se da la presencia social dentro de la red.

Las redes se pueden definir a partir de aspectos individuales o comunitarios, sin embargo, cualquiera que sea la situación, está presente la idea de intercambios o interconexiones entre personas. Estas pueden ser de orden material, servicios, emocionales $\mathrm{u}$ otros acordes a las necesidades de las personas. En general, ninguno funciona de manera aislada. Una primera distinción básica se evidencia entre grupos de apoyos informales y formales:

- La red o el grupo primario es informal. Se basa en las relaciones personales, familiares y comunitarias no estructuradas. La familia se considera una de las formas más comunes de apoyo dentro de las redes sociales, se centra en el afecto y estimula la socialización al satisfacer las necesidades básicas de comunicación e intimidad. Las redes de amistades y vecinos/as son un apoyo valioso para las personas; se construyen por afinidad, intereses comunes 
y actividades compartidas. El sostén que brindan las amistades son más públicas, pero más personales, lo cual es esencial para no sentirse aislada o sola (Méndez \& Cruz, 2008).

La red o el grupo secundario es formal. Sus miembros desarrollan roles concretos, basados en una organización formal y estructurada que considera objetivos específicos en determinadas áreas. Se puede integrar con organizaciones no gubernamentales, instituciones públicas y privadas que brindan solución a demandas sociales específicas y se focalizan en la experiencia colectiva y organizaciones (Méndez \& Cruz, 2008).

De acuerdo con esta distinción entre red primaria y secundaria, podemos afirmar que la presencia social hace referencia a los diferentes intercambios interpersonales que implican afecto, ayuda y afirmación, constituyendo un flujo de intercambio de recursos, acciones e información. Los tipos de apoyo o de intercambios interpersonales, de acuerdo con Guzmán, Huenchuan y Montes (2003), se pueden resumir principalmente en: a) apoyos instrumentales a través de la ayuda, cuidado y acompañamiento; b) apoyos emocionales expresado mediante el cariño, confianza, empatía, sentimientos asociados la preocupación e interés por el otro y c) apoyos cognitivos por medio del intercambio de experiencias, transmisión de información y consejos. Todos estos apoyos, contribuyen a generar un sentimiento de satisfacción, puesto que logran un mayor sentido de control y competencia personal.

Atendiendo a todas estas aportaciones, estudiar la relación existente entre presencia social y las redes sociales online, supone una oportunidad para dar a conocer los beneficios que obtienen las mujeres de zonas rurales, tales como: contactar con otras personas, crear grupos de relación e interés, buscar empleo, crear grupos colaborativos y romper barreras con las que se han ido encontrando las mujeres tradicionalmente, así como conocer la presencia social como indicador para indagar en las políticas de inclusión digital a nivel de género y ruralidad. Por otro lado, la contribución de este estudio radica en que mientras la mayor parte de investigaciones se han centrado en analizar la presencia social en entornos virtuales de aprendizajes (Gunawardena \& Zittle, 1997; Richardson \& Swan, 2003) nosotros nos centramos en entornos abiertos como es el caso de las redes sociales. También encontramos que las investigaciones previas se han focalizado en mayor medida en el análisis de la presencia cognitiva (Busto \& Coll, 2010; Onrubia, Colomina \& Engel, 2008) o la presencia didáctica (Marcelo, 2002; Marcelo \& Perera 2007) y menos en la presencia social, haciéndolo especialmente en contextos escolares o académicos.

\section{Método.}

El estudio se ha desarrollado aplicando el método descriptivo de encuesta con el propósito de conocer el tipo y grado de presencia social existente en las redes sociales usadas por las mujeres de zonas rurales de la provincia de Sevilla. Para ello, el estudio adopta un diseño ex post facto basado en una encuesta, que nos permite conocer el nivel de inclusión digital de estas mujeres en las redes sociales, ofreciendo información real y actual, útil para orientar las políticas de inmersión digital. Este diseño permite conocer y valorar los factores que determinan el uso de las redes sociales así como describir y conocer los contextos y 
condiciones de uso en la práctica. En última instancia, nos permite conocer y valorar los puntos fuertes y débiles del uso que hacen las mujeres de las redes sociales tanto para la mejora de las propias redes como para la mejora de la formación orientada a las mujeres.

\subsection{Participantes.}

La muestra fue seleccionada de entre la población general mediante un muestreo estratificado por edad, y dentro de cada estrato, por cuotas en función del nivel de estudios. Dicha muestra estuvo constituida por 478 mujeres, lo que permitió fijar el error muestral en $\pm 4,57 \%$ para un nivel de significación de $95^{\prime} 5 \%$. Se han considerado como estratos los municipios de la provincia y la edad. En cuanto a la edad, los estratos considerados son: d» 25 años $(24,7 \%), 26$ a 39 años $(32,3 \%), 40$ a 54 años $(32,1 \%)$ y $>55$ años $(10,1 \%)$. En cuanto a los municipios, los estratos considerados han sido las comarcas de la provincia de Sevilla según la siguiente distribución: El Aljarafe (41,21\%), Bajo Guadalquivir (7,11\%), La Campiña $(11,50 \%)$, Sierra Norte $(8,78 \%)$, Sierra Sur $(25,31 \%)$ y Vega del Guadalquivir (6,06\%). Estos municipios atienden, según la Ley para el Desarrollo Sostenible del Medio Rural (2007), a zonas rurales de pequeño tamaño $(<5000$ habitantes), con densidad de población baja o media, empleo diversificado, niveles bajos o medios de renta y alejadas de las ciudades; y zonas rurales periurbanas $(<30000$ habitantes), que están más pobladas, tienen mayoría de trabajadores en el sector terciario, niveles medios o altos de renta y se sitúan cerca de las ciudades.

\subsection{Instrumento.}

Se diseña, aplica y valida una escala de medida de la presencia social de las mujeres en las redes sociales, compuesta por 18 items de tipo Likert con opciones de respuestas expresivas de intensidad $(0=$ nada, $1=$ poco, $2=$ suficiente, $3=$ mucho y $4=$ totalmente) para el autoposicionamiento de las mujeres. Estos indicadores son organizados según las cinco dimensiones teóricas explicadas anteriormente: a) copresencia, b) interdependencia afectiva percibida, c) interdependencia conductual percibida, d) comprensión percibida del mensaje, y; e) dedicación atencional. Tras el análisis de fiabilidad y validez realizado mediante escalamiento óptimo (CATPCA), el procedimiento reliability de SPSS y análisis factoriales, hemos eliminado los ítems 1, 9 y 18 por presentar pesos factoriales inferiores a 0,300 en el primer componente principal, quedando finalmente una escala depurada y válida que está compuesta por los 15 items restantes. Una vez realizado el escalamiento final y cálculo de indicadores, obtenemos una muy alta fiabilidad (Alfa de Cronbach $=0,912$ ) para la escala finalmente obtenida. La unidimensionalidad y validez de constructo viene indicada por los altos coeficientes de saturación de todos los ítems en el primer componente principal (saturación media $=0,652$, con una desviación típica es de 0,154 ) lo que indica la calidad de las medidas.

\subsection{Procedimiento.}

Una vez determinado el tamaño de la muestra y las cuotas según los estratos por edad, se contactó con el área de Cohesión Social e Igualdad de la Diputación Provincial de Sevilla para diseñar la estrategia de 
recogida de información en colaboración con los municipios pertenecientes a las zonas rurales de la provincia y para que hicieran una revisión de la encuesta (contenido, redacción ítems, adecuación vocabulario a la población, etc.). Se estableció un procedimiento para la obtención de información mediante entrevista estructurada, es decir, con presencia de la persona encuestadora. Se sugiere esta forma de aplicación debido a la extensión de la encuesta y a los contenidos de la misma, asegurando la calidad de los datos de cada mujer estudiada.

Para realizar dicho trabajo de campo se contó con la colaboración de las Agentes de Igualdad de los Puntos de Información a la Mujer (PIM) de cada municipio. Estas fueron entrenadas en una sesión formativa grupal donde se les presentó el proyecto, se les dio información sobre los objetivos y metodología del mismo, se les dieron instrucciones para la selección de las mujeres (estratos y cuotas) y sobre la correcta administración de la encuesta. Cada agente de igualdad encuestó a 12 mujeres de cada municipio, con un muestreo selectivo por cuotas, sin reposición, dentro del territorio de su responsabilidad, lo que determinó la consecución de los 478 cuestionarios finales. En todos los casos, las mujeres participantes fueron debidamente informadas del carácter voluntario y anónimo de su participación en el estudio y de los objetivos del mismo, aceptando participar voluntariamente sin recibir ninguna compensación a cambio. Los datos recogidos fueron analizados mediante el paquete estadístico SPSS. Con este software, hemos realizado análisis descriptivos (frecuencias, porcentajes, medidas de tendencia central y dispersión para las variables contempladas en el estudio) de las variables para conocer su estructura y distribución. Para este trabajo presentamos los resultados de Presencia Social (PS) en una escala de tres puntos (poco, suficiente, mucho), una vez comprobada que dicha recodificación no afecta a su validez y fiabilidad. Se crea una variable de cada una de ellas con la puntuación global a partir del sumatorio de todos los ítems, quedando la variable global de PS en una escala de 0 a 60 puntos compuesta por cuatro dimensiones que miden la copresencia, interdependencia afectiva percibida, comprensión percibida del mensaje y dedicación atencional.

\section{Resultados.}

En este apartado presentamos los resultados obtenidos a partir de una escala que hemos elaborado para medir la presencia social de las mujeres en las redes sociales, ya que lo que pretendemos es conocer la percepción que tienen las mujeres de las zonas rurales sobre la presencia social en las redes sociales.

La Tabla y Figura 1, muestran la descripción de la variable «presencia social», donde se han considerado todos los items que componen la escala de tipo Likert, está medida en una escala de 0-60 puntos (sumatorio de los 15 ítems, de 0 a 4 puntos cada uno, cuya suma compone la medida).

Los datos obtenidos indican una media de 21,57 y una desviación típica de 9,892, lo que corresponde con un nivel bajo de presencia social en la red. No obstante, encontramos una variedad en la respuesta que se puede ver en la Figura 1, donde se observa que hay mujeres en cualquier posición de la escala. De hecho, al considerar la elevada desviación típica estamos viendo como hay una dispersión de los datos en torno a casi 10 


\section{Estadísticos}

\begin{tabular}{|ll|r|}
\hline N & Válidos & 451 \\
& Perdidos & 27 \\
Media & & 21,57 \\
Mediana & & 21,00 \\
Moda & 24 \\
Desv. típ. & & 9,892 \\
Mínimo & & 0 \\
Máximo & & 60 \\
\hline
\end{tabular}

Tabla 1. Presencia Social $(0-60)$.

\section{Presencla soclal $(0-60)$}

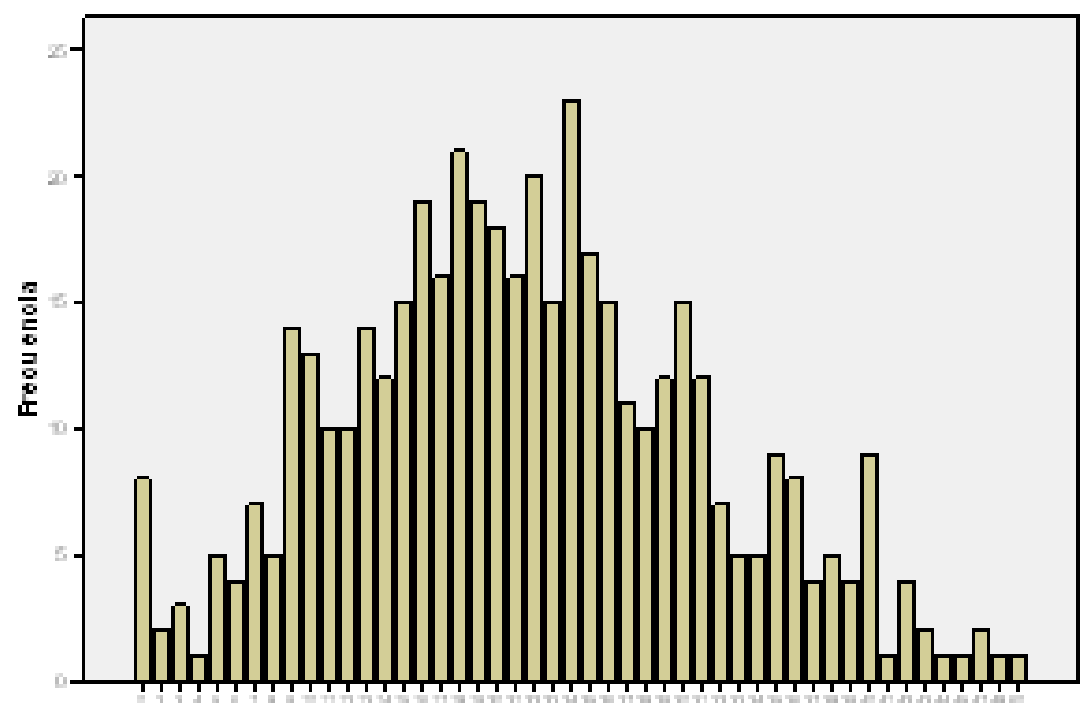

Figura 1. Presencia Social global.

Píxel-Bit. Revista de Medios y Educación. $N^{o} 48$ Enero 2016. ISSN: 1133-8482. e-ISSN: 2171-7966. doi: http://dx.doi.org/10.12795/pixelbit.2016.i48.10 


\begin{tabular}{|l|c|c|}
\hline \multicolumn{1}{|c|}{ Ítem } & M & D.T. \\
\hline Mi red social me ayuda a sentirme integrada en sociedad & 2,13 & 1,116 \\
\hline Mi estado de ánimo mejora al usar mi red social & 2,13 & 1,116 \\
\hline Me expreso claramente en mi red social para que los demás & 2,41 & 1,251 \\
me entiendan & & 1,052 \\
\hline Comprendo las ideas y sentimientos que expresan las otras & 2,45 &, 977 \\
\hline Las personas de mi red social me prestan atención & 2,13 & \\
\hline
\end{tabular}

Tabla 2. Descriptivos de algunos indicadores de PS.

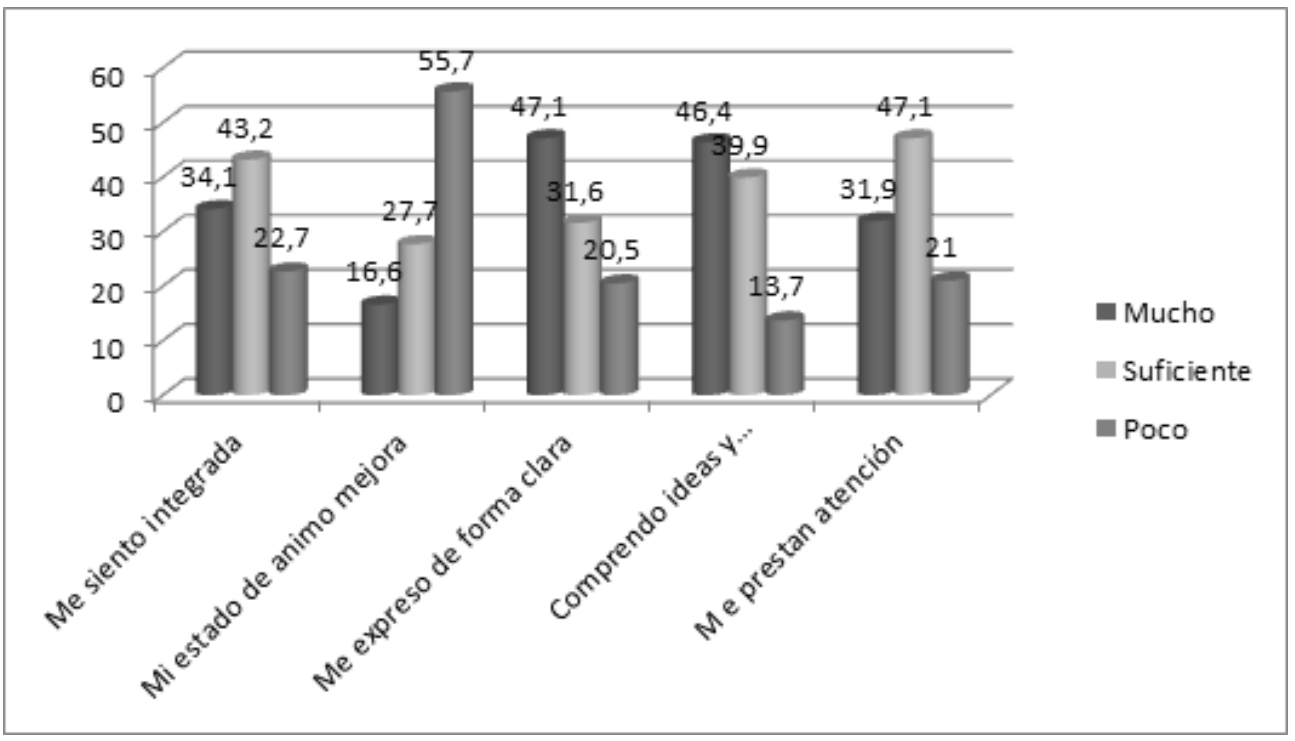

Figura 2. Distribución de mujeres en algunos indicadores de PS. 
puntos, indicando dicha variabilidad interna en este concepto.

Del conjunto de aspectos de la Presencia Social que exploramos en este estudio, las mujeres señalan cinco aspectos como los más percibidos y, por tanto, los que mejor definen su percepción de la presencia social en las redes: mi red me ayuda a sentirme integrada, el estado de ánimo mejora con su uso, me expreso claramente en mi red social para ser entendida, comprendo las ideas y sentimientos de las personas con las que interactúo, recibo atención de las personas de mi red social. La Tabla 2 muestra las medidas de tendencia central y dispersión en estos ítems.

Como se observa en la Figura 2, entre un $77,3 \%$ y $79 \%$ de mujeres perciben un nivel medio-alto de presencia social en estos indicadores, sin embargo un grupo de mujeres que oscila entre un $21 \%$ y un $22,7 \%$ no encuentra presencia social en las redes sociales.

En contraste con estos aspectos, encontramos que el $77,3 \%$ de las mujeres que han respondido a los constructos relacionados con la consciencia de presencia de otras personas en su red social, junto a la capacidad de comprender y expresar los sentimientos y emociones a través de la red, -además de sentirse escuchadas y atendidas por las personas con las que interactúan-, fomentan un entorno virtual que promueve los sentimientos de cohesión y compromiso con las otras personas (Blanchard, 2007; Herrero \& Gracia, 2007). Estos datos concuerdan con los estudios de Agut et al. (2011), que afirman que cuando las personas se sienten conectadas con su grupo, no se sienten solas en el entorno y cuentan con la posibilidad de expresarse, compartir opiniones o solicitar ayuda a otras personas, es decir, perciben que están más integradas socialmente. Asimismo, investigaciones como las de Garrison, Anderson y Archer (2000) y Rourke et al. (2001), ponen de manifiesto que los principales indicadores que afectan a la percepción de la presencia social son el componente afectivo, relacionado con la identidad del propio grupo, la motivación y la cohesión, incentivadores todos ellos del sentimiento de integración. En efecto, mecanismos como la motivación, comunicación, participación e interactividad son variables que hemos considerado en nuestro estudio como principales indicadores para mejorar la participación, la interactividad entre la comunidad en línea y el establecimiento de lazos entre las personas que componen el grupo.

Por otro lado, hemos identificado con un $30,3 \%$ que los constructos relacionados con la interdependencia afectiva y conductual no son considerados esenciales por las mujeres para proyectarse a sí mismas a través de las redes sociales de manera social y emocional. Esto podría ser indicio de que las mujeres tienen mayor facilidad para conectar con otras personas en el plano cognitivo que en el emocional, lo cual podría ayudar a explicar por qué las puntuaciones de los ítems relacionados con estos constructos han sido más bajos. Esto puede afectar al tipo y grado de interacción creada a través de las redes sociales.

Entendemos que dichos resultados podrían orientar el diseño de las redes sociales, siendo la presencia social uno de sus ejes centrales. Así, en la medida en que estos entornos faciliten la interacción con otras personas (familia, amigos/as compañeros/as...) de una forma sencilla, cercana y real, fomentarán una mayor participación y colaboración. Ignorar estas cuestiones afectaría negativamente a 
la motivación e implicación en las redes sociales.

\section{Discusión.}

Este estudio nos ha permitido constatar que las mujeres de zonas rurales perciben un nivel medio de presencia social en las redes sociales. La mayoría de ellas afirman que le influye nada o poco la red en su estado de ánimo ni en su comportamiento, mientras que su presencia es suficiente o mucha en lo que se refiere a sentirse integrada, escuchada y comunicada con otras personas.

La interdependencia afectiva y conductual no son considerados por las mujeres como elementos esenciales para proyectarse a través de las redes sociales. Esto indica que las mujeres tienen mayor facilidad para conectar con otra persona en el plano cognitivo que en el emocional. Esta diferencia refleja la desconfianza que aún siguen provocando estos tipos de herramientas comunicativas, por lo que sería necesario establecer pautas de actuación enfocadas a la mejora de la expresión emocional a través de las redes sociales online, para fortalecer el tipo y grado de interacción creada.

Estos resultados sugieren un efecto positivo de las políticas de inclusión digital en el acceso y uso funcional de las mujeres de los entornos rurales analizados. Parece importante continuar con estas políticas con el fin de estimular usos rutinizados y avanzados de las tecnologías en general, y de las redes sociales en particular, en las mujeres del mundo rural como un medio para promover la ciudadanía digital, procurando la mayor cohesión y equidad social y atendiendo a grupos que, por sus especiales características, pueden encontrarse en mayor riesgo de exclusión, siendo las mujeres del medio rural un colectivo de especial atención. Nuestro estudio indica también que muchas mujeres no han recibido formación sobre el uso de redes, sugiriendo que su aprendizaje se vincula a contextos no formales y a redes personales. Esto sugiere la necesidad de repensar las posibles estrategias de formación adoptadas en relación con las mujeres adultas de zonas rurales. En este sentido, algunas líneas de actuación en las que se tendría que incidir para que exista una mayor presencia social en las redes sociales online serían:

- Conocer otros procesos psicosociales que pueden emerger en estos entornos y su relación con la presencia social, así como, explorar si la relación entre la percepción de presencia social y el uso de las redes es estrictamente lineal o de otro tipo.

Modificar el diseño de las redes sociales, teniendo la presencia social como uno de sus ejes centrales. Así, en la medida en que estos entornos faciliten la interacción con otras personas de una forma sencilla, cercana y real, fomenten una mayor participación y colaboración.

Fortalecer el establecimiento de redes de mujeres intergeneracionales que mejoren el nivel de la presencia social y cooperación entre ellas.

Fomentar actividades de formación enfocadas a una mayor implicación afectiva con respecto al uso y manejo de las redes sociales, con el fin de visualizar sus beneficios a nivel psicológico y emocional. 


\section{Referencias bibliográficas.}

Agut, S., Grandío, A. \& Lozano, F. (2011). Presencia social en entornos virtuales de aprendizaje: Adaptación al español de la Networked Minds Social Presence Measure. Revista Latinoamericana de Psicologia, 43 (2), 279-288. doi: http://dx.doi.org/10.14349/ rlp.v43i2.172

Anderson, T. D., Rouke, L., Garrison, D. R. \& Archer, W. (1999). Social presence in computer conferencing: Chewing the phat(ic). Unpublished paper.

Biocca, F., Harms, C. \& Burgoon, J. K. (2003). Toward a more robust theory and measure of social presence: Review and suggested criteria. Presence: Teleoperators and Virtual Environments, 12(5), 456-480. doi: 10.1162/105474603322761270

Blanchard, A. (2007). Developing a sense of virtual community measure. CyberPsychology \& Behavior, 10 (6), 82730. doi: 10.1089/cpb.2007.9946

Busto, A. \& Coll, C. ( 2010). Los entornos virtuales como espacios de enseñanza y aprendizaje. Una perspectiva psicoeducativa para su caracterización y análisis. Revista Mexicana de Investigación Educativa, 44 (15), 163-184.

Garramone, G. M., Harris, A. C. \& Anderson, R. (1986). Uses of political computer bulletin boards. Journal of Broadcasting \& Electronic Media, 30, 325-339. doi: 10.1080/ 08838158609386627

Garrison, D. \& Anderson, T. (2005). El elearning en el siglo XXI. Investigación y práctica. Barcelona: Octaedro.

Garrison, D. R. \& Archer, W. (2001). A transactional perspective on teaching and learning: A framework for adult and higher education. Oxford, UK: Pergamon Presence.
Garrison, D. R., Anderson, T. \& Archer, W. (2000). Critical inquiry in a text-based environment: Computer conferencing in higher education. The Internet and Higher Education, 2 (2), 87-105. doi: http:// dx.doi.org/10.1016/S1096-7516(00)00016-6

Garrison, D. R., Anderson, T. \& Archer, W. (2001). Critical thinking, cognitive presence, and computer conferencing in distance education. American Journal of Distance Education, 15 (1), 7-23.

Gunawardena, C. N. (1995). Social presence theory and implications for interaction and collaborative learning in computer conferences. International Journal of Educational Telecommunications, 1, 147166.

Gunawardena, C. N. (2003). Social presence and the sociocultural context of online education. Recuperado de http:// a of 20 . an adolu.edu.tr/bildiriler/ Charlotte_Lani.doc

Gunawardena, C. \& Zittle, F. (1997). Social Presence as a Predictor of Satisfaction within a Computer-mediated Conferencing Environment. The American Journal of Distance Education, 11 (3), 8-26. doi: 10.1080/ 08923649709526970

Guzmán, J., Huenchuan, S. \& Montes, V. (2003). Redes de apoyo social de las personas mayores. Marco conceptual, Revista Notas de Población de la CEPAL, 77, CELADE División de Población de la CEPAL.

Harms, C. \& Biocca, F. (2004). Internal consistency and reliability of the networked minds social presence measure. En M. Alcañiz \& B. Rey (Eds.), Seventh Annual International Workshop. Presence 2004. Universidad Politécnica de Valencia. 
Herrero, J. \& Gracia, E. (2007). Measuring perceived community support: Factorial structure, longitudinal invariance, and predictive validity of the PCSQ (Perceived Community Support Questionnaire). Journal of Community Psychology, 35 (2), 197-217. doi: 10.1002/jcop.20143

Kerr, E. B., \& Hiltz, S. R. (1982). Computermediated communication systems: Status and evaluation. New York: Academic Press.

Kreijns, K. (2004). Sociable CSCL environments. Social affordances, sociability and social. Cyberpsychology \& behavior, 7 (2), 155-172.

Ley 45/2007 de 13 diciembre para el Desarrollo Sostenible del Medio Rural. Boletin Oficial del Estado, Madrid, España, 14 de diciembre de 2007. Recuperado de http:/ /www.boe.es/buscar/pdf/2007/BOE-A-200721493-consolidado.pdf

Limious, M. \& Smith, M. (2010). Teachers' and students'perspectives on teaching and learning through virtual learning environments. European Journal of Engineering Education, 35 (6), 645-653. doi:10.1080/03043797.2010.505279

Marcelo, C. (2002). Aprender a enseñar para la sociedad del conocimiento, Educational Policy Analysis Archives, 10 (35).

Marcelo, C. \& Perera V. H. (2007). Comunicación y aprendizaje electrónico: la interacción didáctica en los nuevos espacios virtuales de aprendizaje. Revista de Educación, 343, 381-429.

Méndez, E. y Cruz L. (2008). Redes sociales de apoyo y persona adulta mayor: Costa Rica. Documento preparado para el I Informe estado de situación de la persona adulta mayor en Costa Rica, UCR/ CONAPAM.
Onrubia, J., Colomina, R. \& Engel, E. (2008). Los entornos virtuales de aprendizaje basados en el trabajo en grupo y el aprendizaje colaborativo. En C. Coll \& C. Monereo (eds.), Psicología de la educación virtual. Enseñar y aprender con las tecnologías de la información y la comunicación (pp. 233-252). Madrid: Morata.

Perse, E. I., Burton, P., Kovner, E., Lears, M. E. \& Sen, R. J. (1992). Predicting computermediated communication in a college class. Communication Research Reports, 9 (2), 161 170. doi: DOI:10.1080/08824099209359908

Richardson, J. \& Swan, K, (2003). Examining social presence in online courses in relation to students' perceived learning and satisfaction. Journal of Asynchronous Learning Networks, 7 (1), 68-88.

Rourke, L., Anderson, T., Garrison, R. \& Archer, W. (2001). Assessing Social Presence in asynchronous text-based computer conferencing. Journal of Distance Education, 14 (1), 1-18.

Santos, G. (2011). Presencia social en foros de discusión en línea. Píxel-Bit. Revista de Medios y Educación, 39 (1), 17-28.

Serrano, Y. (2005). Assessing faculty's social presence indicators in online courses at the InterAmerican University of Puerto Rico, Bayamón Campus. Recuperado de http:// bc.inter.edu/focus/a4_n1/yolanda.pdf.

Short, J., Williams, E. \& Christie, B. (1976). The social psychology of telecommunications. New York: John Wiley \& Sons.

Tu, C. H. \& Mcisaac, M. S. (2002). An examination of social presence to increase interaction in online classes. American Journal of Distance Education, 16 (2), 131150. 
Uzunboylu, H., Bicen, H. \& Cavus, N. (2011). The efficient virtual learning environment: A case study of Web 2.0 tools and Windows live spaces. Computers and Education, 56 (3), 720-726. doi: 10.1016/ j.compedu.2010.10.014

Fecha de recepción: 27-04-2015

Fecha de evaluación: 31-05-2015

Fecha de aceptación: 16-06-2015

Píxel-Bit. Revista de Medios y Educación. $N^{o} 48$ Enero 2016. ISSN: 1133-8482. e-ISSN: 2171-7966. doi: http://dx.doi.org/10.12795/pixelbit.2016.i48.10 